\title{
A mathematical model of the immune system's role in obesity- related chronic inflammation
}

Authors: Pablo Díaz ${ }^{a}$, Michael Gillespie ${ }^{b}$, Justin Krueger ${ }^{c}$, José Pérez ${ }^{d}$, Alex Radebaugh ${ }^{e}$, Toby Shearman ${ }^{f}$, Garret Vo ${ }^{g}$, Christine Wheatley ${ }^{h}$

${ }^{a}$ University of North Carolina at Greensboro, ${ }^{b}$ St. Augustine's College, ${ }^{c}$ Miami University, ${ }^{d}$ University of Puerto Rico, Mayagüez Campus, ${ }^{e}$ Bucknell University, ${ }^{f}$ Virginia Tech, ${ }^{g}$ Montana State University, ${ }^{h}$ Alma College

Contact Information: Justin Krueger, Phone: 513-519-2663， Email: justimk@cinci.rr.com

Sponsors: J. Bassaganya-Riera ${ }^{i}$, J. Borggaard ${ }^{j}$, J. Burns ${ }^{j}$, E. Cliff $^{j}$, A. Guri ${ }^{i}$, S. Faulkner ${ }^{i}$, R. Hontecillas-Magarzo ${ }^{i}$, A. Jarrah ${ }^{i}$, C. Koelling ${ }^{j}$, R. Laubenbacher ${ }^{i}$, H. Mortveit ${ }^{i}$, L. Zietsman $^{j}$

${ }^{i}$ Virginia Bioinformatics Institute at Virginia Tech

${ }^{j}$ Interdisciplinary Center for Applied Mathematics at Virginia Tech

Contact Information: Dr. Reinhard Laubenbacher, Phone: 540-231-7506, Email: reinhard@vbi.vt.edu

\section{Abstract}

Obesity is quickly becoming a pandemic. The low-grade chronic inflammation associated with obesity leads to health risks such as cancer, heart disease, and type 2 diabetes mellitus. To better understand the progression of obesity-related chronic inflammation, mice were fed either a high-fat or low-fat diet over 140 days. At Days 0, 35, 70, and 140, the percentages of macrophage subsets, CD4 $+\mathrm{T}$ cells, and regulatory $\mathrm{T}$ cells infiltrating the intra-abdominal white adipose tissue (WAT) were examined. Monocyte chemoattractant protein-1 (MCP-1) mRNA expression in WAT was also quantified. Additionally, glucosenormalizing ability was examined by administering peritoneal glucose tolerance tests. A system of ordinary differential equations models this system. The model consists of 8 differential equations, has 25 parameters, and has 1 forcing function. Tools used to characterize the model include parameter estimation, sensitivity analysis, and stability analysis. Based on the data provided, the system describes the growth of adipocyte size and chronic inflammation over 105 days beginning at Day 35, which is approximately when the adipose cells become hypertrophic, or too large to function normally. The model shows that without intervention, chronic inflammation escalates and the related health problems persist. 


\section{Introduction}

Obesity, which is defined as a body mass index over 30, continues to grow as a health concern in the United States [5]. As recently as July 2008, the Centers for Disease Control reported a phone survey that shows approximately 26 percent of Americans over the age of 18 are obese [5]. Other research shows that the prevalence of obesity has increased by 70 percent over the past decade [10]. According to World Health Organization estimates, over 300 million adults are obese [12]. As the severity of the problem continues to grow worldwide, many scientific experts consider the obesity crisis a pandemic [11].

Low-grade chronic inflammation is currently believed to be the most probable link between obesity and its co-morbidities. Unlike acute inflammation, which is the natural response to injury or infection, chronic inflammation results from a defective immune response. The excessive activity of pro-inflammatory cells and proteins can result in additional defects for surrounding tissues. Chronic inflammation can lead to diseases such as cancer, kidney failure, atherosclerosis, and type 2 diabetes mellitus [2].

When a body is obese, the adipocyte cells swell to abnormal volumes. As the adipocytes continue to enlarge, the cells' endoplasmic reticulum comes under excessive stress and fails. This leads to the excessive production of pro-inflammatory proteins such as monocyte chemoattractant protein-1 (MCP-1) and tumor necrosis factor- $\alpha$ (TNF- $\alpha$ ) [2]. MCP-1 is a chemokine that attracts cells expressing the $\mathrm{C}-\mathrm{C}$ motif chemokine receptor 2 (CCR2) on their cell surface [13]. This is relevant because recent studies have shown that the migration of macrophages expressing the glycoprotein F4/80 and CCR2 at high concentrations is associated with impaired glucose tolerance [6].

The release of TNF- $\alpha$ and MCP- 1 and the subsequent progression of chronic inflammation are shown in Figure 1 below.

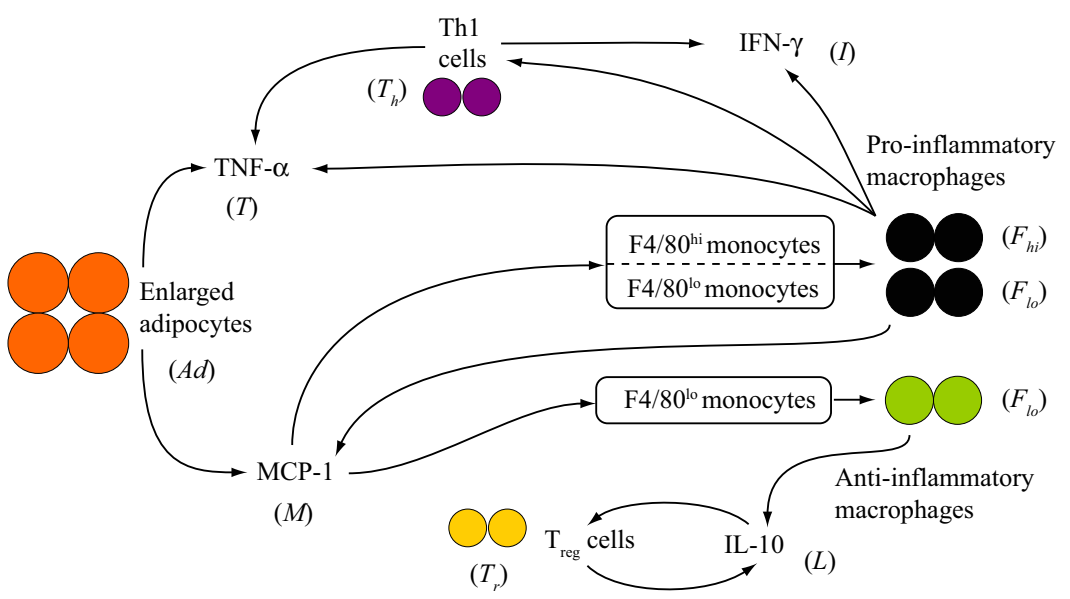

Figure 1: Diagram of Chronic Inflammation [2].

The monocytes that reach the white adipose tissue interact with various cytokines to differentiate into 4 characterized subsets of macrophages. The four subsets are M1, M2, deactivated, and transforming growth factor- $\beta$ (TGF- $\beta$ ). M1 and deactivated macrophages are 
the most relevant subsets to the system. M1 macrophages are induced by the presence of interferon- $\gamma$ (IFN- $\gamma$ ) and secrete interleukin-12 (IL-12), MCP-1, and TNF- $\alpha$. Deactivated macrophages are induced by interleukin-10 (IL-10) and also secrete IL-10 [16].

$\mathrm{T}$ cells are also drawn to the adipose tissue. Many of the $\mathrm{T}$ cells are already differentiated into pro-inflammatory and anti-inflammatory cells once they reach the adipose tissue, but a significant portion of the differentiation is due to the same cytokines that convert monocytes to macrophages. The interaction between naïve T cells and IL-12 produces pro-inflammatory T helper (Th1) cells. The Th1 cells produce IFN- $\gamma$, more MCP-1, and more TNF- $\alpha$, which further contribute to the inflammation. Similarly, the interaction between naïve $\mathrm{T}$ cells and IL-10 produce anti-inflammatory T regulatory ( $\mathrm{Treg}$ ) cells. The Treg cells manufacture and release more IL-10 [1].

The role that chronic inflammation plays in health issues such as obesity-related insulin resistance are well documented [15]. There are numerous other mathematical models related to the immune system and chronic inflammation. Some models describe the effect of the immune system on type 1 diabetes [3] [9] while other models describe inflammation resulting from shock or trauma [14]. The focus on obesity-induced chronic inflammation and the number of incorporated immune system components separate this model from other models of the immune system. Also, the potential to include insulin resistance seen in type 2 diabetes mellitus and possible drug treatments make this initial study valuable for understanding and eventually controlling the diabetes pandemic.

This research aims to aid and complement current studies on chronic inflammation by building a mathematical model that accurately reflects the interactions of the macrophages, $T$ cells, chemokines, and cytokines that cause chronic inflammation. The model provides quantitative insight into the progression of chronic inflammation that would not otherwise be possible due to the limits of data collection.

\section{Model/Methods}

\subsection{Experiment and Data}

Drs. Josep Bassaganya-Riera and Amir Guri performed all the experiments and collected all the data used for this research in their laboratory at the the Virginia Bioinformatics Institute at Virginia Tech.

A collection of C57BL/6J mice were separated into three test groups: high-fat diet, lowfat diet, and control. The mice were put in identical environments that prevented any opportunities for exercise, so there would be no variations other than their diet. At Day 0 the control group was necropsied; on Days 35, 70, and 140 of the experiment mice on high-fat and low-fat diets were also necropsied.

The percentage of macrophage subsets, $\mathrm{CD} 4+\mathrm{T}$ cells, and regulatory $\mathrm{T}$ cells in the white adipose tissue (WAT) were collected using flow cytometry. MCP- 1 and TNF- $\alpha$ gene expression in the WAT were measured using real-time RT-PCR. Finally, glucose-normalizing ability was examined using a peritoneal glucose tolerance test. 
The amount of available data varies depending on the species being viewed. Averages are taken for the four time points for the mice on the high-fat diet. In addition, the Day 35 data is interpolated using an unweighted average of Day 0 and Day 70 for Th1, Treg and MCP-1 because data does not exist. Initially, we have a total of four average data points for MCP-1, T cells and macrophages; however, the scope of our model decreases the data to three reference points, Days 35, 70 and 140.

Having the cell data as a percentage of the total cells in the stromal-vascular fraction (SVF) poses a problem because the data reflects any changes in the rest of the SVF, which the model cannot possibly do. Our model assumes the change in the number of stromalvascular cells not considered in the model to be zero and converts between the data given as percentages and cell counts to solve the system, which presents an additional assumption.

\subsection{System of Equations}

The model has nine variables and twenty-five parameters. The system consists of eight differential equations, six of which are linear. A ninth equation characterizes the forcing function. Some agents that contribute to obesity-induced inflammation are not accounted for in this model. For our system we only considered the following factors:

Table 1: Variables and associated factors.

\begin{tabular}{cl}
\hline \hline Variable & Associated Factor \\
\hline$A d$ & Adipocyte size \\
$T$ & Concentration of TNF- $\alpha$ \\
$M$ & Concentration of MCP-1 \\
$F_{h i}$ & Population of F4 $/ 80^{\mathrm{hi}} \mathrm{CCR} 2^{+}$macrophages \\
$F_{l o}$ & Population of F4/80 ${ }^{\mathrm{lo}} \mathrm{CCR} 2^{+}$macrophages \\
$T_{h}$ & Population Thelper 1 cells \\
$I$ & Concentration of IFN- $\gamma$ \\
$T_{r}$ & Population of Treg cells \\
$L$ & Concentration of IL-10 \\
\hline \hline
\end{tabular}

We consider Day 35, the approximate time for average adipocyte hypertrophy, as the beginning of our model. Adipocytes secrete TNF- $\alpha$ and MCP-1. TNF- $\alpha$ is a cytokine that increases insulin resistance, and $\mathrm{MCP}-1$ plays a significant role in the recruitment of monocytes expressing CCR2 on their surface. The monocytes that MCP-1 recruits to the site are classified as high or low, corresponding to the expression of F4/80 on the cell surface. The F4/80 hi monocytes $\left(F_{h i}\right)$ only become pro-inflammatory macrophages and the $\mathrm{F} 4 / 80^{\mathrm{lo}}$ monocytes $\left(F_{l o}\right)$ are uncharacterized, producing either pro or anti-inflammatory macrophages [2]. Pro-inflammatory macrophages contribute to the production of IFN$\gamma$, a cytokine that is also secreted by Th1 cells. Anti-inflammatory macrophages secrete IL-10, which is also released by Treg cells. From this overview, the system of equations 
seen below is created. In the system, cells are measured as populations, and protein concentrations are measured in gene expressions (pg-cDNA/ $\mu \mathrm{g}-\mathrm{RNA})$. The adipocyte size is measured by the cross-sectional area $\left(\mu \mathrm{m}^{2}\right)$ of the adipocytes.

$$
\begin{aligned}
\frac{d T(t)}{d t} & =k_{1}\left(F_{h i}(t)+\alpha F_{l o}(t)\right)+k_{2} A d(t)+k_{3} T_{h}(t)-d_{1} T(t) \\
\frac{d M(t)}{d t} & =k_{4}\left(F_{h i}(t)+\alpha F_{l o}(t)\right)+k_{5} A d(t)-d_{2} M(t) \\
\frac{d F_{h i}(t)}{d t} & =k_{6}\left(M(t) I(t)-\frac{\alpha M(t) L(t)}{1-\alpha}\right)-d_{3} F_{h i}(t) \\
\frac{d F_{l o}(t)}{d t} & =k_{7} M(t) L(t)+k_{8} \frac{\alpha M(t) L(t)}{1-\alpha}-d_{4} F_{l o}(t) \\
\frac{d T_{h}(t)}{d t} & =k_{9}\left(F_{h i}(t)+\alpha F_{l o}(t)\right)+m_{1}-d_{5} T_{h}(t) \\
\frac{d I(t)}{d t} & =k_{10} T_{h}(t)+k_{11}\left(F_{h i}(t)+\alpha F_{l o}(t)\right)-d_{6} I(t) \\
\frac{d T_{r}(t)}{d t} & =k_{12} L(t)+m_{2}-d_{7} T_{r}(t) \\
\frac{d L(t)}{d t} & =k_{13} T_{r}(t)+(1-\alpha) k_{14} F_{l o}(t)-d_{8} L(t) \\
A d(t) & =a \sqrt{t}+b
\end{aligned}
$$

We consider all of the F4/80 hi macrophages to be pro-inflammatory. However, the F4/ $80^{\text {lo }}$ macrophages are uncharacterized, so we define $\alpha$ to be the fraction of $\mathrm{F} 4 / 80^{\mathrm{lo}}$ macrophages that are pro-inflammatory.

MCP-1 attracts monocytes with CCR2 receptors; therefore, we assume that given a nonzero concentration of MCP-1 there are monocytes available in the adipose tissue. For the purpose of this model, the representative interaction between MCP-1 and IFN- $\gamma$ differentiates the monocytes into pro-inflammatory macrophages, and the representative interaction between MCP-1 and IL-10 differentiates the monocytes into anti-inflammatory macrophages. Our model must be able to differentiate between the $\mathrm{F} 4 / 80^{\mathrm{hi}}$ and the $\mathrm{F} 4 / 80^{\mathrm{lo}}$ pro-inflammatory macrophage production. There exists some fraction $\beta$ of the indirect interaction between MCP- 1 and IFN- $\gamma$ that produces $\mathrm{F} 4 / 80^{\mathrm{lo}}$ macrophages. Thus, $(1-\beta)$ is the fraction of the indirect interaction that leads to $\mathrm{F} 4 / 80^{\text {hi }}$ macrophages. Using the definition of $\alpha$ we can say

$$
\frac{\beta M I}{(\beta M I+M L)}=\alpha .
$$


Solving for $\beta M I$ gives

$$
\beta M I=\frac{\alpha M L}{(1-\alpha)} .
$$

The right-hand side of this equation can then be substituted for $\beta$ MI, the number of $\mathrm{F} 4 / 80^{\text {lo }}$ pro-inflammatory macrophages, in any of the differential equations, eliminating the need for another parameter.

Equation (1) represents the rate of change in the concentration of TNF- $\alpha$ with respect to time. TNF- $\alpha$ is initially released by the enlarged adipocytes in the system, although the main production of this cytokine is accredited to pro-inflammatory macrophages [2]. The term $\left(F_{h i}(t)+\alpha F_{l o}(t)\right)$ describes the total number of pro-inflammatory macrophages. Th1 cells also produce TNF- $\alpha$. The concentration of TNF- $\alpha$ decreases due to its denaturation.

Equation (2) represents the change in concentration of MCP-1 with respect to time. MCP-1 is also initially released by the enlarged adipocytes in the system. Once monocytes become M1 macrophages, however, the activated macrophages, presumably F4/80 hi CCR2 ${ }^{+}$ macrophages, contribute to $\mathrm{MCP}-1$ concentrations [6].

The change in the population of $\mathrm{F} 4 / 80^{\text {hi }}$ macrophages with respect to time is represented by Equation (3). The first term accounts for the production of all $\mathrm{F} 4 / 80^{\mathrm{hi}}$ macrophages while the second term determines the number of pro-inflammatory macrophages that are $\mathrm{F} 4 / 80^{\mathrm{lo}}$. The third term accounts for their death.

The change in the population of $\mathrm{F} 4 / 80^{\mathrm{lo}}$ is represented in Equation (4). The first term accounts for the interaction of $\mathrm{F} 4 / 80^{\mathrm{lo}}$ monocytes with IL-10 to produce anti-inflammatory $\mathrm{F} 4 / 80^{\mathrm{lo}}$ macrophages. The second term, like in Equation (3), represents the F4/80 ${ }^{\mathrm{lo}}$ monocytes that become pro-inflammatory macrophages. The third term accounts for macrophage death.

In Equation (5), the pro-inflammatory macrophages release IL-12, which differentiates naïve $\mathrm{T}$ cells into Th1 cells. The presence of pro-inflammatory macrophages, $\left(F_{h i}(t)+\right.$ $\left.\alpha F_{l o}(t)\right)$ implies the presence of IL-12 since pro-inflammatory macrophages produce IL12. For simplicity, we take advantage of this relationship and assume that the Th1 cell population is dependent on pro-inflammatory macrophages. The term $m_{1}$ accounts for any migration of Th1 cells to the site, naturally occurring or by chemokines and cytokines we are not considering in our model. Finally, $d_{5} T_{h}(t)$ represents the removal or death of Th1 helper cells.

Th1 cells and pro-inflammatory macrophages produce IFN- $\gamma$, so they directly increase the concentration of IFN- $\gamma$. The IFN- $\gamma$ concentration decreases with its denaturation or internalization by its receptor, which is shown in Equation (6).

In regard to Equation (7), IL-10 increases the concentration of adaptive $T$ regulatory cells by differentiating naïve $\mathrm{T}$ cells. The term $m_{2}$ accounts for any migration of $\mathrm{T}$ regulatory cells to the site. Finally, $d_{7} T_{r}(t)$ represents the death of T regulatory cells.

T regulatory cells and anti-inflammatory macrophages secrete IL-10 during the immune response. The concentration decreases through denaturation or internalization by its receptor, as shown in Equation (8). 
Adipocyte size (Ad) has a logarithmic correlation with body weight, for which we have data [4]. Equation (9) represents the enlargement of adipocytes with respect to time.

\subsection{Parameter Estimation}

Our model includes 25 parameters. Fourteen of these parameters describe interaction rates, eight describe component degradation, two describe cell migration rates, and one

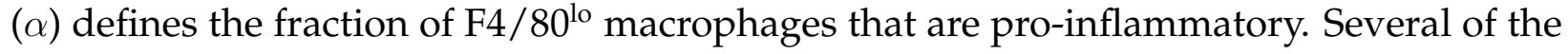
parameters may be amenable to special laboratory observations; many other parameters only make sense within the context of model equations (1)-(9). Because experimental procedures isolating each effect are impractical, we are led to mathematical methods to characterize good parameter values.

To evaluate the quality of a parameter set, we quantitatively describe the error of the corresponding solution against available data using the 2-norm - the distance between the solution and the data. Due to concerns about the accuracy of the MCP-1 data, the calculation ignores the MCP-1 data even though the data is plotted in Figure 2(b). The 2-norm computes the errors using the percentage of total cells for $\mathrm{F} 4 / 80^{\mathrm{hi}}$ and $\mathrm{F} 4 / 80^{\mathrm{lo}}$ macrophages, Th1 cells, and T regulatory cells at Days 35, 70, and 140. The equation for the error $J(p)$ is below.

$$
J(p)=\frac{1}{2} \sum_{k}\left\|x\left(t_{k} ; p\right)-\hat{x}\left(t_{k}\right)\right\|^{2}
$$

Defining an appropriate range for each parameter is important for implementing a random search method for which we write an algorithm. As the model is written, all parameters are defined to be positive. Unfortunately, we have no further knowledge of the interaction parameters ranges; therefore, these were selected in the range $[0,0.1]$ in order to maintain reasonable cell populations. The values for $k_{6}, k_{7}$, and $k_{8}$ are set equal due to their similar nature. Based on equilibrium constraints of equations (5) and (7), functions of $d_{5}$ and $d_{7}$, respectively, determine the parameters $m_{1}$ and $m_{2}$. Once inside the adipose tissue, macrophages survive on the order of months, so the range for the degradation of $\mathrm{F} 4 / 80^{\mathrm{hi}}$ and $\mathrm{F} 4 / 80^{\mathrm{lo}}$ macrophages is $[0,1]$. We expect the proteins and other immune cells to live on the order of hours and days, respectively, and their initial ranges are set accordingly. Finally, we set $\alpha$ equal to zero since the latest experimental data shows a very high percentage, if not all, of the $\mathrm{F} 4 / 80^{\mathrm{lo}}$ macrophages are anti-inflammatory.

We implemented a solver for the system of eight coupled, differential equations using one of MATLAB's built-in ODE solvers. Since the cell variables grow much faster than the gene expression variables, the system (1)-(9) is stiff. Therefore, we used MATLAB's stiff equation solver (ode15s).

Three optimization methods were used to decrease the error and to better match the system solution to the data: a random search method, a Nelder-Meade unconstrained minimization procedure ( $\mathrm{fminsearch}$ ), and a constrained, nonlinear minimization algorithm ( $f$ mincon), which is part of the MATLAB Optimization Toolbox.

The initial conditions are experimental values from Day 35. After selecting a parameter set which minimizes the error and adheres to the constraints, we allow the initial conditions to vary, along with all 25 parameters. Adjusting the initial conditions improves the 
overall-fit. We select the following set of initial conditions and parameters to characterize our model:

Table 2: Final Values for the 8 Initial Conditions, $\mathrm{x}_{0}$.

\begin{tabular}{cccl}
\hline \hline State & Value $^{*}$ & State & \multicolumn{1}{c}{ Value $^{*}$} \\
\hline$T$ & $2.74 \times 10^{-5}$ & $M$ & $1.12 \times 10^{-5}$ \\
$F_{h i}$ & $1.176 \times 10^{1}$ & $F_{l o}$ & $1.331 \times 10^{1}$ \\
$T_{h}$ & $1.679 \times 10^{1}$ & $I$ & $3.4 \times 10^{-5}$ \\
$T_{r}$ & $2.968 \times 10^{0}$ & $L$ & $7.270 \times 10^{-5}$ \\
\hline
\end{tabular}

${ }^{*}$ Cell values are cell populations (in thousands) and protein values are measured in pg-cDNA/ $\mu \mathrm{g}$-RNA.

Table 3: Final Values for the Parameter Set, p.

\begin{tabular}{cccl}
\hline \hline Parameter & Value & Parameter & Value \\
\hline$k_{1}$ & $7.10 \times 10^{-9}$ & $k_{8}$ & $1.71 \times 10^{10}$ \\
$k_{2}$ & $4.22 \times 10^{-8}$ & $k_{9}$ & $6.82 \times 10^{-7}$ \\
$k_{3}$ & $5.81 \times 10^{-8}$ & $k_{10}$ & $7.66 \times 10^{-6}$ \\
$k_{4}$ & $5.70 \times 10^{-14}$ & $k_{11}$ & $1.16 \times 10^{-5}$ \\
$k_{5}$ & $6.88 \times 10^{-9}$ & $k_{12}$ & $1.60 \times 10^{4}$ \\
$k_{6}$ & $1.42 \times 10^{10}$ & $k_{13}$ & $1.04 \times 10^{-4}$ \\
$k_{7}$ & $1.07 \times 10^{10}$ & $k_{14}$ & $9.11 \times 10^{-6}$ \\
$d_{1}$ & 6.289 & $d_{5}$ & 1.472 \\
$d_{2}$ & 2.474 & $d_{6}$ & 7.700 \\
$d_{3}$ & 0.569 & $d_{7}$ & 0.628 \\
$d_{4}$ & 0.842 & $d_{8}$ & 5.798 \\
$m_{1}$ & 23.44 & $m_{2}$ & 0.586 \\
$\alpha$ & 0.0 & \multicolumn{3}{c}{} \\
\hline$a^{*}$ & $3.421 \times 10^{2}$ & $b^{*}$ \\
\hline \hline
\end{tabular}

Note that the largely different values of the collected data (cell counts and gene expressions), as discussed before, produce a staggering range for the parameter set. Parameter values associated with the interactions of MCP-1 with other proteins (which both have experimental values on the order of $10^{-5}$ ) will have parameter values on the order $10^{10}$. Likewise, parameter values associated with the production of proteins by cells will have values on the order of $10^{-10}$ or often smaller because a large number of cells are producing relatively small concentrations of proteins. Scaling the units of the parameters may improve the aesthetics, but likely would not change the results. 


\section{Results}

After deciding upon a specific set of parameters, we numerically solve the system of equations. Each subsequent solution is graphed independently as seen below. The solution is obtained using one of MATLAB's ${ }^{\mathrm{TM}}$ built-in ODE solvers. Several plots show the average data points to which the solution is fitted, while the remaining solutions are a product of the system.

Figure 2(a) shows that from Day 35 to Day 140, the gene expression for TNF- $\alpha$ increases at a moderate rate.

From Day 35 to Day 140, the gene expression for MCP-1 gradually increases (Figure 2(b)). As previously explained, the average data points for MCP-1 appear in the figure, but the solution is independent of the data because the data is ignored due to accuracy concerns. We chose to include the data in the figure to illustrate that data was collected for MCP-1.

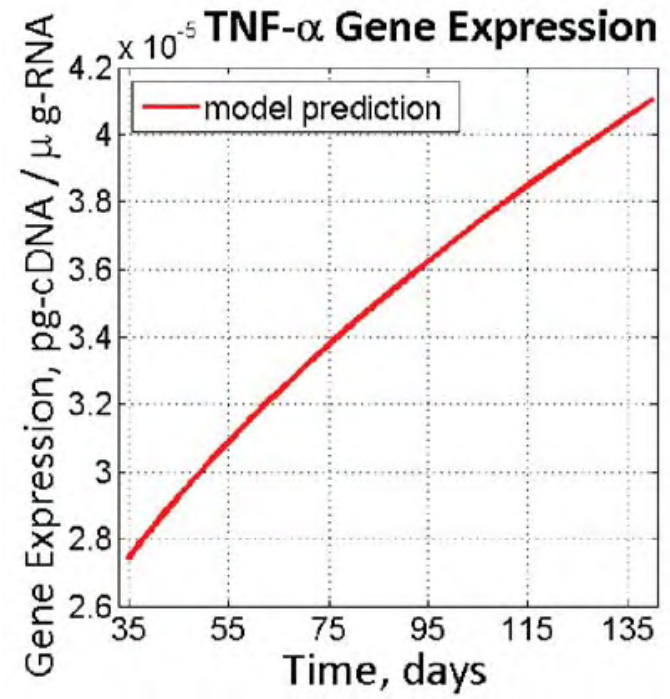

(a) TNF- $\alpha$ gene expression.

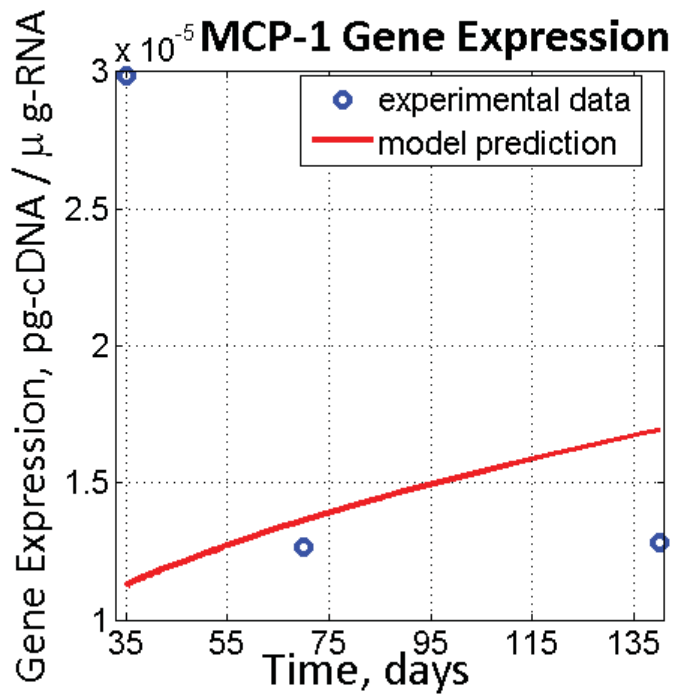

(b) MCP-1 gene expression.

Figure 2: Results of the gene expressions of TNF- $\alpha$ and MCP-1.

The percentage of F4/80hi macrophages immediately decreases, followed by a sharp increase over the 105-Day period in which adipocytes are hypertrophic. The solution, seen in Figure 3(a), is shown to fit the data with moderate error as the line goes through one data point and is in close proximity to the Day 70 and Day 140 averages.

The percentage of $\mathrm{F} 4 / 80^{\mathrm{lo}}$ macrophages is shown to follow the same general trend as the $\mathrm{F} 4 / 80^{\text {hi }}$ macrophages, steeply decreasing followed by a significant increase in the last 95 days (Figure 3(b)). The percentage of F4 $/ 80^{\mathrm{lo}}$ macrophages at Day 140 is slightly smaller than the percentage of F4 $/ 80^{\text {hi }}$ macrophages at the same time. The error is also relatively small between the solution and plotted data points. 


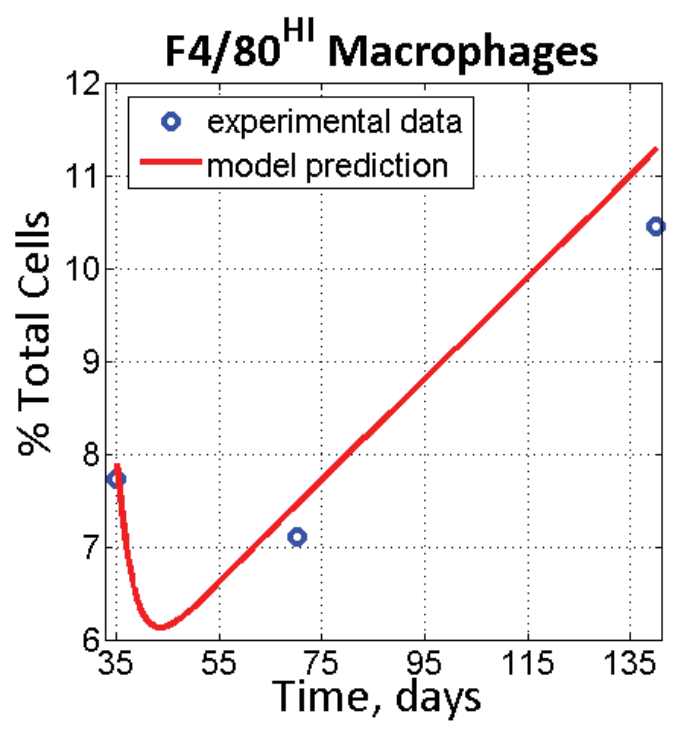

(a) F4/80 mi macrophages.

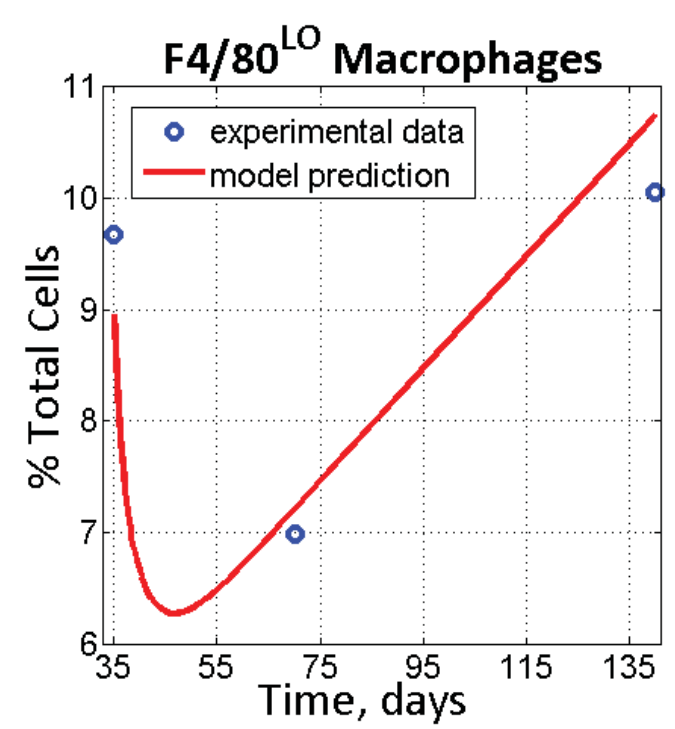

(b) F4/80 lo macrophages.

Figure 3: Results of the populations of F4/80 hi and F4/80 lo macrophages.

The percentage of Th1 cells decreases slightly, possibly due to the rapid decline of the cell population to equilibrium. This is followed by a quick increase and finally a steady decline for the 105-day period, which is shown in Figure 4(a). The data points show similar trends to the solution, but the change in magnitude between the average data points is much greater. Therefore, the error is relatively larger for this solution.

Figure 4(b) shows an initial sharp decrease occurs in the gene expression of IFN- $\gamma$ succeeded by a gradual increase in gene expression.

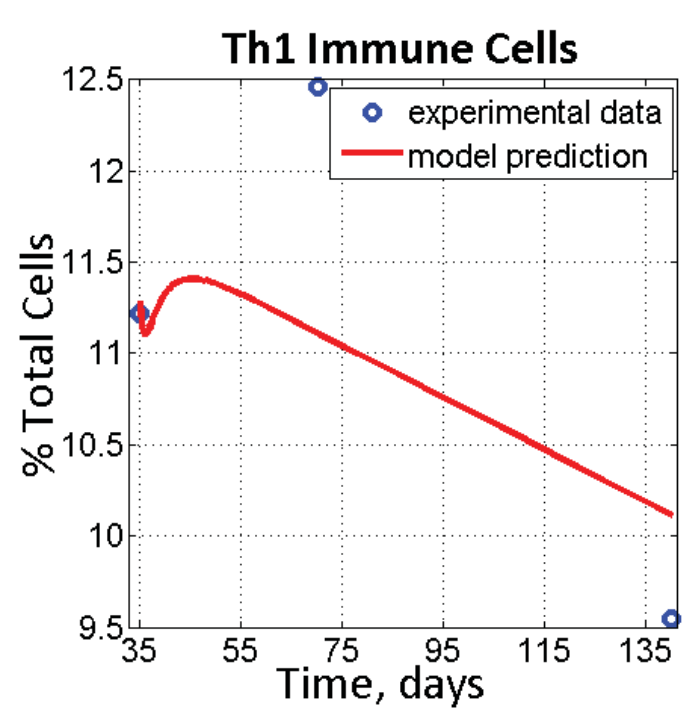

(a) Th1 immune cells.

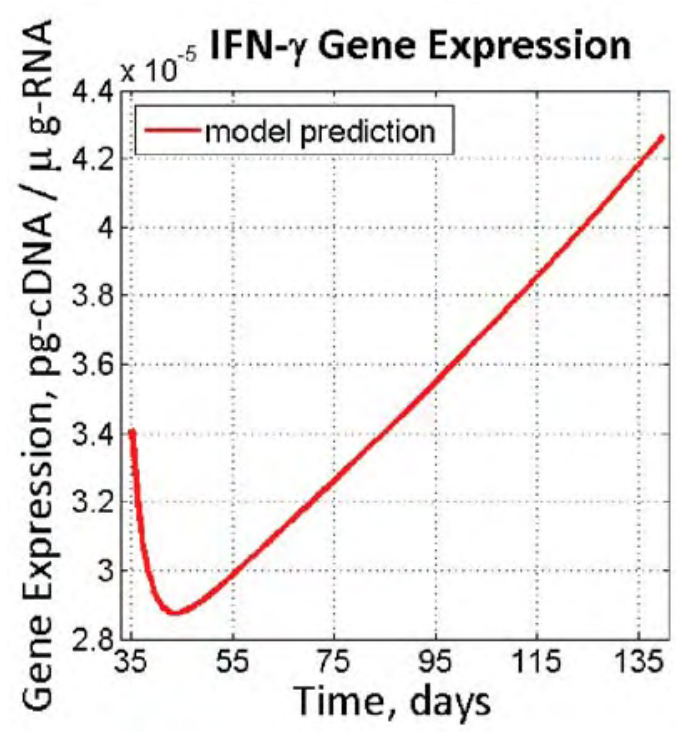

(b) IFN- $\gamma$ gene expression.

Figure 4: Results for the population of Th1 cells and the expression of IFN- $\gamma$. 
Considering total cells in the system, the percentage of $\mathrm{T}$ regulatory cells quickly decreases and then slowly increases (Figure 5(a)). The error is small as the solution comes close to the three data points.

The gene expression for IL-10 decreases at a fast rate and then sharply increases for the remaining 90-day period as seen in Figure 5(b).

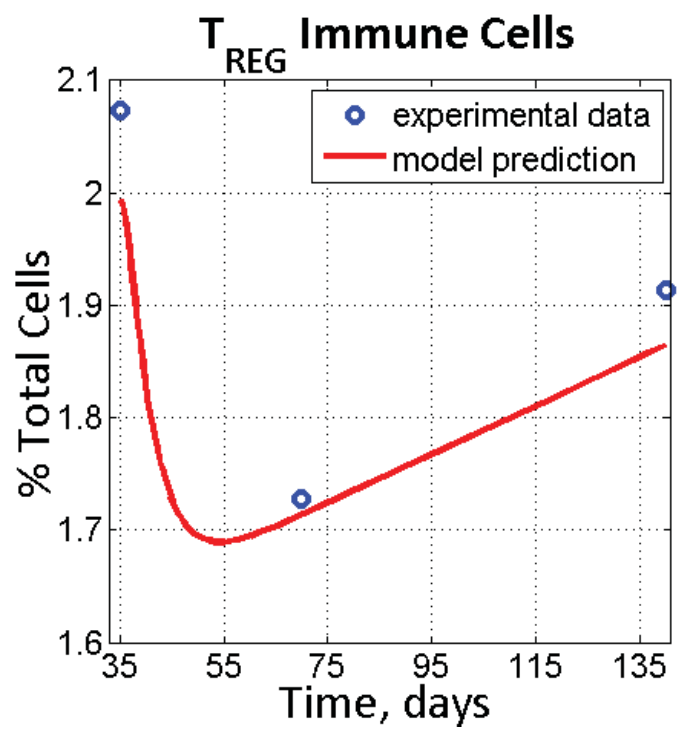

(a) Treg immune cells.

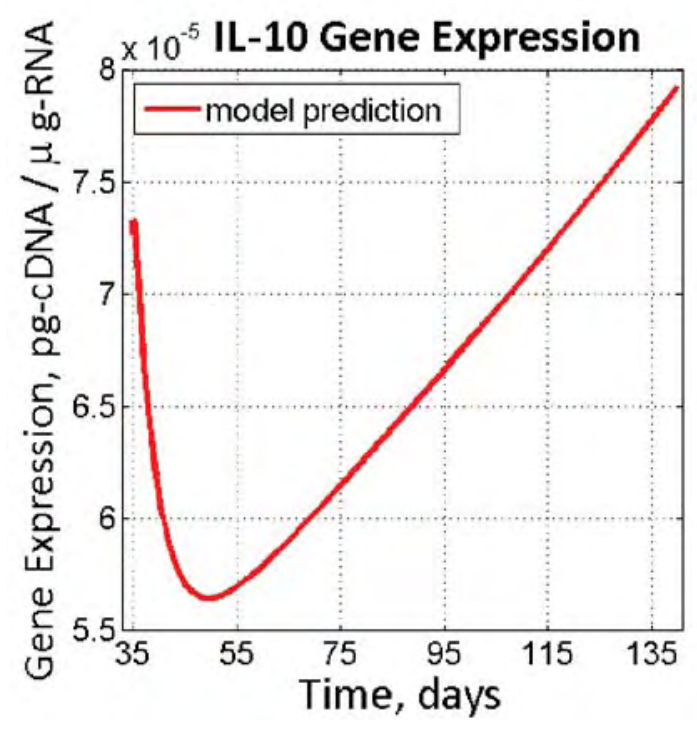

(b) IL-10 gene expression.

Figure 5: Results for the population of Treg cells and the expression of IL-10.

\subsection{Sensitivity Analysis}

Understanding how the solution changes with respect to the parameters' changes can give insight into the biology. Therefore, analyzing how sensitive the model is with respect to the parameters becomes important. We describe the sensitivities mathematically by

$$
S_{i}^{j}=\frac{\partial x_{i}}{\partial p_{j}},
$$

where $i$ represents a particular variable (1)-(8), and $j$ signifies the particular parameter (1)-(25). The matrix $\left[S_{i}^{j}\right]$ consists of 200 entries and is time dependent.

We compute the sensitivities of our nonlinear system by solving the coupled state/sensitivity initial value problem. Define $\bar{S}^{j}(t)$ by summing the sensitivities over each of the $i$ (state) values, $\bar{S}^{j}(t)=\sum_{i=1}^{8}\left|S_{i}^{j}\right| . \quad \bar{S}^{j}(t)$ provides insight into how each parameter affects the system as a whole, at a given time, $t$. As seen Figure 6 , sensitivity analysis helps identify important parameters like $k_{5}, d_{2}$, and $d_{8}$. Figure 6 depicts the system's sensitivities to each parameter, summed over the eight states and integrated from Day 35 to 140. In terms of $\bar{S}^{j}(t)$, Figure 6 shows $\int_{35}^{140} \bar{S}^{j}(t) \mathrm{d} t$ for each of the $j$ (parameter) values. 


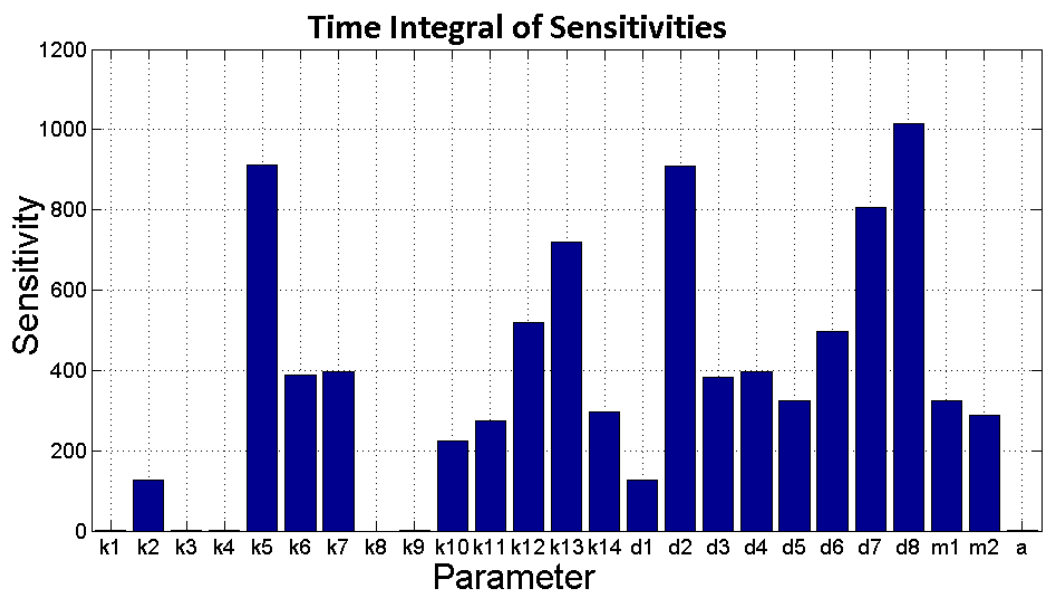

Figure 6: Sensitivities with respect to each of the 25 parameters summed over the statespace and integrated with respect to time.

Each of the sensitivities has been scaled by both their respective parameter value and the average value of the state over Days 35 to 140 to understand the relative sensitivities. Moreover, it is important to recognize that these are local values. The sensitivities depend on the area of existence in the parameter and time spaces; with different parameter sets and at different times the sensitivities may change drastically. Taking parameters $k_{12}$, $k_{13}$, and $k_{14}$ as examples, Figure 7 emphasizes the locality of parameter sensitivities with respect to time.

Figures like 7 and 8 reveal more information than Figure 6 and provide a more complete understanding of the system.

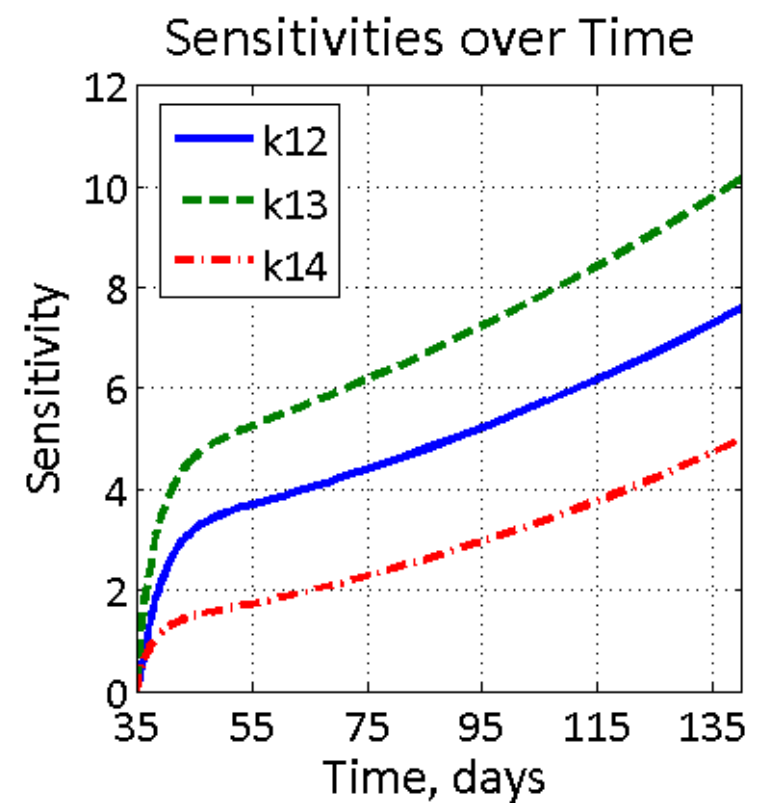

Figure 7: Sensitivities of the system to $k_{12}, k_{13}$, and $k_{14}$ summed over the state-space, emphasizing the change with respect to time. 
To further understand the impact and importance of these sensitivities in the parameter estimation process, consider the gradient of the cost function:

$$
J(p)=\frac{1}{2} \sum_{k}\left\|x\left(t_{k} ; p\right)-\hat{x}\left(t_{k}\right)\right\|^{2}
$$

in which $k$ represents the time steps where data was gathered, and $\hat{x}$ denotes the experimental data set. Differentiating with respect to the parameters gives:

$$
\frac{\partial J(p)}{\partial p_{j}}=\sum_{k}\left[\left\|x\left(t_{k} ; p\right)-\hat{x}\left(t_{k}\right)\right\| \cdot \frac{\partial x\left(t_{k}\right)}{\partial p_{j}}\right] .
$$

The gradient of the cost function contains $\frac{\partial x_{i}}{\partial p_{j}}$, which are the sensitivities. For nonzero error terms, $\left[x\left(t_{k} ; p\right)-\hat{x}\left(t_{k}\right)\right]$ in the equation, the corresponding sensitivities drive that error's contribution to the gradient. Hence, highly sensitive parameters control large changes in the cost function. The gradient and an understanding of the sensitivities will help in implementing a more sophisticated parameter optimization technique.

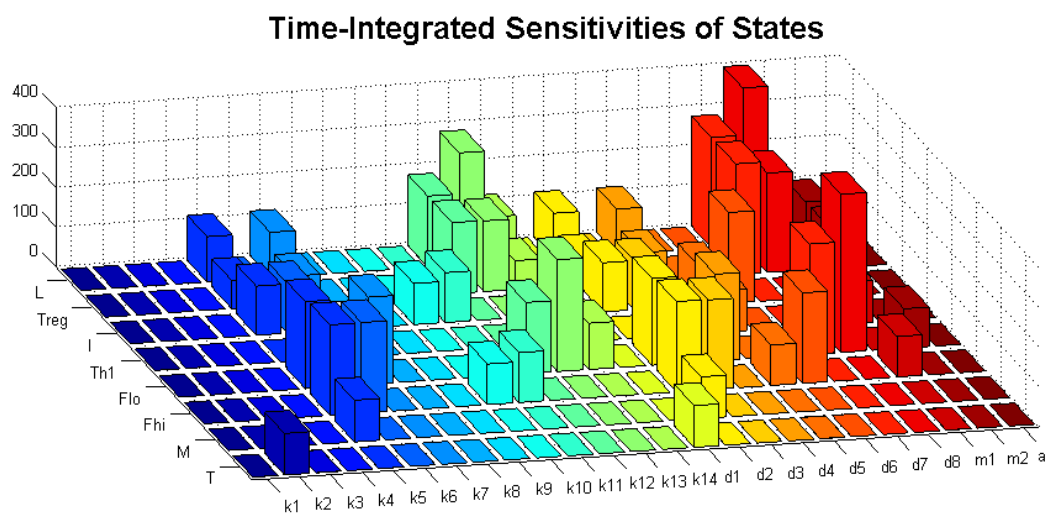

Figure 8: Sensitivities of each of the 8 variables with respect to each of the 25 parameters, integrated with respect to time.

\subsection{Stability Analysis}

The adipocyte size is held constant throughout the stability analysis at $4069 \mu \mathrm{m}^{2}$, the Day 35 value [4]. The six linear differential equations have the change in each state, the lefthand side, set equal to zero, and the $\mathrm{F} 4 / 80^{\mathrm{hi}}, \mathrm{F} 4 / 80^{\mathrm{lo}}$, and adipocyte terms are subtracted to the left hand side. The system of equations can now be written and solved using matrices. The solution gives the six linear differential equations written in terms of $\mathrm{F} 4 / 80^{\mathrm{hi}}$, $\mathrm{F} 4 / 80^{\mathrm{lo}}$, and adipocyte size. The three variables in the $\mathrm{F} 4 / 80^{\mathrm{hi}}$ and $\mathrm{F} 4 / 80^{\mathrm{lo}}$ equations - MCP-1, IFN- $\gamma$, IL-10 - are replaced by their respective equations written in terms of $\mathrm{F} 4 / 80^{\mathrm{hi}}$ and $\mathrm{F} 4 / 80^{\mathrm{lo}}$ and the left-hand sides of these two equations are set to zero. The system's equilibrium is now condensed down to two implicit functions.

The F4 $/ 80^{\mathrm{hi}}$ and F4 $/ 80^{\mathrm{lo}}$ equations are plotted on the same graph. Intersections between the two curves indicate where the entire system is in equilibrium. The values for F4/80 ${ }^{\text {hi }}$ 
and $\mathrm{F} 4 / 80^{\mathrm{lo}}$ are determined from the plot, and these values are put back into the six equations to determine the values for the remaining six variables. To determine the stability of the system, we look at the eigenvalues of the Jacobian matrix for the entire system of equations.

The two equilibrium points found are analyzed to determine the stability. One equilibrium point gives all negative eigenvalues, and the point is stable. The values are in Table 3. However, the second equilibrium point has one positive eigenvalue and is unstable [8]. In addition, the $\mathrm{F} 4 / 80^{\mathrm{hi}}$ value at the unstable equilibrium point is too high to be biologically valid.

Table 4: Stable Equilibrium Solution.

\begin{tabular}{|c|c|c|}
\hline Variable & Equilibrium Value $^{*}$ & Eigenvalue \\
\hline TNF- $\alpha$ & $2.746 \times 10^{-5}$ & -6.289 \\
\hline MCP-1 & $1.132 \times 10^{-5}$ & -7.953 \\
\hline $\mathrm{F} 4 / 80^{\mathrm{hi}}$ & 7.781 cells & -6.294 \\
\hline $\mathrm{F} 4 / 80^{\mathrm{lo}}$ & 7.579 cells & -2.474 \\
\hline Th1 & 15.931 cells & -0.317 \\
\hline IFN- $\gamma$ & $2.757 \times 10^{-5}$ & -0.200 \\
\hline Treg & 2.274 cells & -0.775 \\
\hline IL-10 & $5.269 \times 10^{-5}$ & -1.472 \\
\hline Ad Size & $4069 \mu \mathrm{m}^{2}$ & $\mathrm{~N} / \mathrm{A}$ \\
\hline
\end{tabular}

\section{Discussion}

One objective was to develop a better sense of the role F4/80 hi macrophages and F4/80 ${ }^{\text {lo }}$ macrophages play in the progression of chronic inflammation. As seen in Figures 3(a) and $3(\mathrm{~b}), \mathrm{F} 4 / 80^{\mathrm{hi}}$ and $\mathrm{F} 4 / 80^{\mathrm{lo}}$ macrophages, respectively, more accurately fit the data when compared to the other six variable solutions. The solution for the percentage of $\mathrm{F} 4 / 80^{\mathrm{lo}}$ macrophages is similar in shape to the solution for percent $\mathrm{F} 4 / 80^{\mathrm{hi}}$ macrophages. However, $\mathrm{F} 4 / 80^{\mathrm{lo}}$ increases at a slower rate, which can be seen more easily when the model extends beyond 140 days. As inflammation worsens, it is expected for pro-inflammatory macrophages to be greater in number than anti-inflammatory macrophages. The solution shows a greater percentage of $\mathrm{F} 4 / 80^{\mathrm{hi}}$ macrophages than $\mathrm{F} 4 / 80^{\mathrm{lo}}$ macrophages at Day 140 , which can be viewed as a faster production rate in the inflamed area.

The TNF- $\alpha$ gene expression (Figure 2(a)) increases as expected because its producers are also increasing. The hypertrophic adipocytes and increasing F4/80 hi macrophages contribute to the increase seen in TNF- $\alpha$. While Th1 cells also produce TNF- $\alpha$, they are not causing the increase in concentration because they are constant in cell count, as explained later.

The plot for MCP-1 gene expression (Figure 2(b)) shows the expected increase in MCP-1 concentration due to the increasing number of pro-inflammatory macrophages and the 
presence of hypertrophic adipocytes in the system. Since the solution for MCP-1 is independent of the three data points, it does not show the very large drop that occurs in MCP-1 gene expression in the first 35-day period. However by observing the solutions, this decrease in the data could correlate to the drop in the number of $\mathrm{F} 4 / 80^{\mathrm{hi}}$ macrophages during the same time period. Discrepancies lie in the period from Day 70 to Day 140 where the solution shows a moderate increase and the raw data shows a fairly constant $\mathrm{MCP}-1$ gene expression. One would predict a greater increase in MCP-1 as the F4/80 hi macrophages drastically increase.

Figure 9, below, shows very unique behavior of Th1 cells in the system. Regardless of the initial condition, the Th1 cell count stabilizes quickly to a constant population. Since the Th1 cell population remains constant, the percentage of Th1 will vary according to the rest of the system. As the macrophages decrease, the Th1 percentage naturally increases and finally when the macrophage percentage increases, the Th1 percentage will decrease. The nature of the Th1 cells needs to be further explored as it is surprising to see a constant cell count indicating the possibility of a missing component in Equation (5).

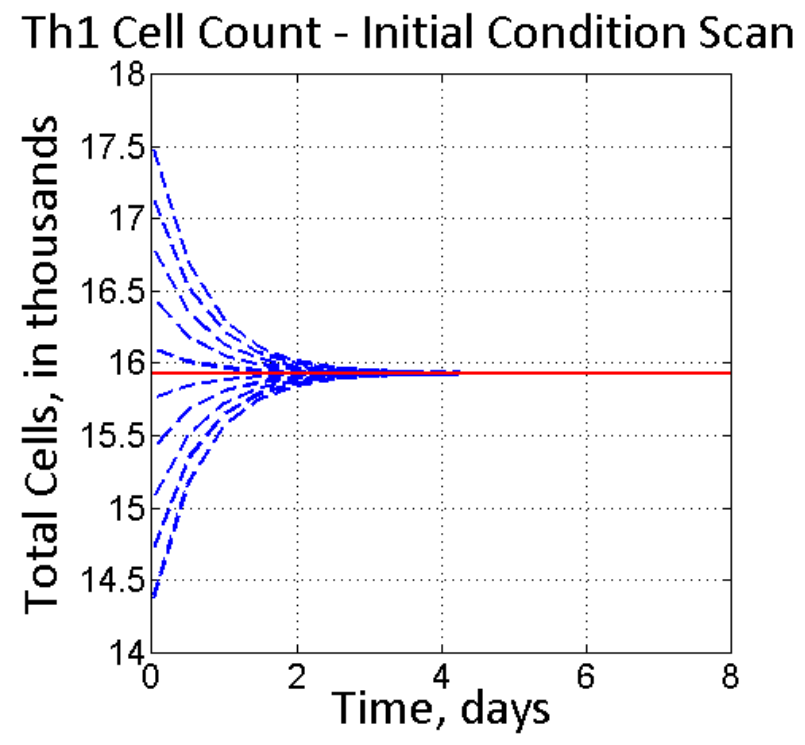

Figure 9: Th1 cell population with varying initial conditions.

Since IFN- $\gamma$ is produced by Th1 and F4/80hi macrophages and Th1 cells are constant in our system, pro-inflammatory macrophages are responsible for the changes in IFN- $\gamma$ concentration.

The Treg solution (Figure 5(a)) is somewhat surprising considering the solution for the percentage of Th1 cells. Because chronic inflammation is dominated by pro-inflammatory molecules, the percentage of $\mathrm{T}_{\text {reg }}$ cells is expected to increase at a slower rate than the Th1 cells. The solution shows, even though the percentage of Treg decreases from Day 35 to Day 140, the percentage decreases less than the percentage of Th1 cells.

The solution for IL-10 gene expression (Figure 5(b)) correlates to the F4/80 ${ }^{\text {lo }}$ macrophage solution. Since F4/80 10 macrophages produce IL-10 it is fitting for the IL-10 solution to 
mimic that of the F4/ $80^{\mathrm{lo}}$ macrophage curve. The IL-10 solution also correlates well with the $\mathrm{T}_{\text {reg }}$ solution because $\mathrm{T}_{\text {reg }}$ cells produce IL-10.

Limitations exist and assumptions must be identified when observing the model. The first issue lies in the data used to select the parameters and to judge the validity of the model. As discussed in the Model and Methods section, the sparsity of data as well as the units of the data both add challenges to the modeling.

The design of the model reflects the interactions of the macrophages, $\mathrm{T}$ cells, chemokines, and cytokines that cause chronic inflammation, after the onset of adipocyte hypertrophy. The model does not account for the time period in which the subject becomes obese. Using body weight data for our study of interest by Guri et al., we track average adipocyte sizes over a period of 140 days, and note Day 35 as being a threshold for problematic adipocyte hypertrophy [7]. Hence the Day 0 data was not used in our model. In addition, the MCP-1 data acquired was questionable due to the sporadic nature of the results. When finding parameters, we are only confident in using the F4/80 ${ }^{\mathrm{hi}}, \mathrm{F} 4 / 80^{\mathrm{lo}}$, Th1 and Treg data for our base platform. This constitutes an over-parameterized problem decreasing the confidence in our initial conditions and model and leaving ample room for improvement in the future when more data is available.

The model must be confined within some set of biological boundaries. The limited scope of the model almost certainly accounts for some of the discrepancies between the data and the model, but the immune system encompasses many systems in the body along with various other proteins and cell types. We ignored many of these components in this model to simplify a complex problem. Immune cells, such as monocytes, travel throughout the body via blood and lymph, but our model isolates the specific destination of these immune cells, the white adipose tissue due to the well-established significance of intraabdominal fat inflammation. The F4/80 macrophages, $\mathrm{T}_{\text {reg, }}$, Th1 cells and specific corresponding proteins, IL-10, IFN- $\gamma$, TNF- $\alpha$, and MCP-1 are extracted from the SVF as focal points for our model. All other components of the SVF are assumed to remain constant in our model.

Ultimately a model should reasonably project into the future, but this model is designed for the small 105-day experimental period of adipocyte hypertrophy. As seen by Figure 10, expanding the time span causes the model to fail. 


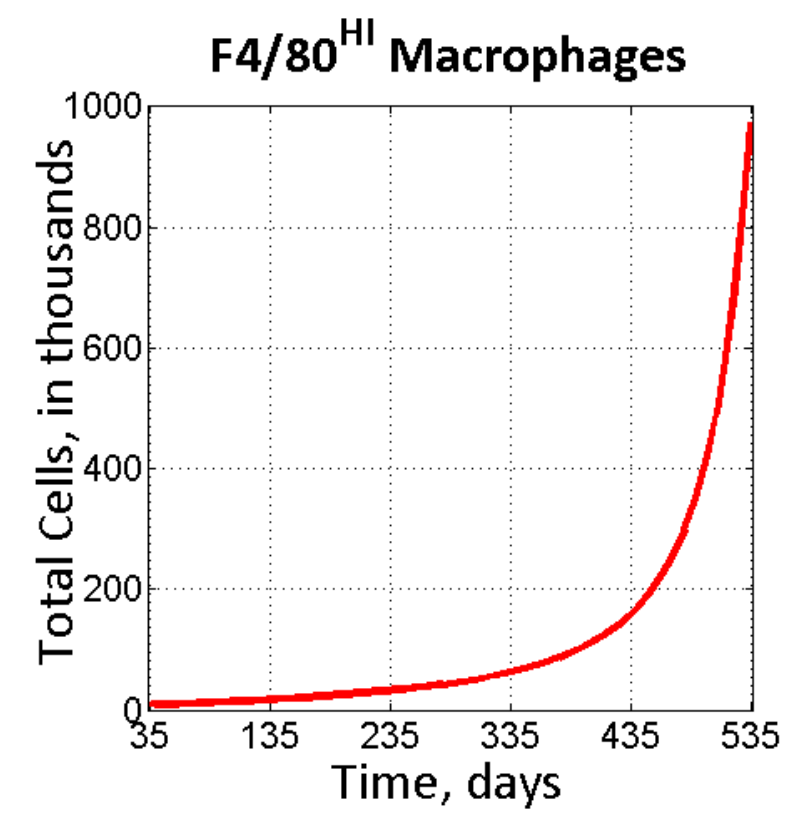

Figure 10: F4/80hi macrophage population.

At about 535 days, the $\mathrm{F} 4 / 80^{\text {hi }}$ macrophages count grows to nearly 1 million cells, which is an unreasonable increase from the initial condition of about 10 cells. We must adjust the system of equations in order to apply the model beyond Day 140.

\section{Conclusion and Future Work}

With obesity continuing to grow as a pandemic, national governments such as the United States have established initiatives to reduce the number of obese citizens over a certain time frame [5]. This makes understanding obesity-related inflammation both timely and urgently needed.

We have developed a mathematical model to quantify the molecular and cellular interactions of chronic inflammation. We fit the solution for the model to the experimental data. The F4 $/ 80^{\text {hi }}$ macrophages increase at a steep rate. The $\mathrm{F} 4 / 80^{\mathrm{lo}}$ macrophages and T regulatory cells also increase but at a lesser rate than the $\mathrm{F} 4 / 80^{\text {hi }}$ macrophages. The chemokine and cytokine levels all also increase which is expected due to the increase in the numbers of cells that produce these proteins. One consequence of higher cytokine levels, for example, is increased TNF- $\alpha$ levels have been linked to increased insulin resistance [1].

The solution also presents two major discrepancies from the expected results: the percentage of Th1 cells decreases, and in looking at the cell count, the Th1 population rapidly decreases to an equilibrium where it resides for the remainder of the time. The other unexpected result is the initial decrease in the percentages and cell populations for both macrophage types and both $T$ cell types.

The limitations and assumptions of the model allow for improvements and further work on the continuous model. The model's simulation capabilities start at Day 35, approxi- 
mately when adipocyte hypertrophy sets in. Extending the model to include Day 0 information will improve the biological relevance of the model. Understanding how the adipose cells reach hypertrophy and the immune system's response in the first 35 days is just as important as understanding what occurs in the adipose tissue after the adipocytes reach hypertrophy.

Similarly, extending the model to accurately reflect the progression of chronic inflammation beyond 140 days would improve the model's value. While the current model has the capability of simulating beyond 140 days, the accuracy of the results are questionable since there is no data for extended time spans currently available to validate the model. The ability to project the changes in chronic inflammation over an experimental subject's lifespan makes the model easier to apply for future research. In addition, having a model that is valid over extended periods of time reduces the necessity of experimental research to gain valuable insight into a biological system.

Another suggestion for further work is to examine the existence of equilibrium at the point in time where adipocytes stop enlarging and the average adipocyte size remains constant. This research analyzes the equilibria that occur once the adipocyte reaches hypertrophy, but the existence of equilibrium at the end of adipocyte swelling is much more relevant. If there is a stable equilibrium where average adipocyte size remains constant, the model says chronic inflammation increases a finite level even without intervention. This knowledge would be applicable to research on both the escalation and treatment of chronic inflammation.

A highlight of future work is adding the effect of drug treatments to the model, which also promises to give a better and more relevant system of equations. Similarly, finding a way to incorporate the relationship between inflammation and insulin resistance would be extremely beneficial. If the model could accurately reflect drug treatments' role in chronic inflammation, a better understanding of how chronic inflammation progresses could result. The drug treatment model could also lead to new ideas for research both in the function of the immune system and in the drugs used to treat chronic inflammation. The research that could stem from the system of equations modeling capabilities could lead to newer, more effective drugs that treat chronic inflammation with fewer side effects.

Our model and the corresponding solution have positive results. The solution reflects what is known to occur as chronic inflammation progresses. The solution does, however, present some questions about the validity of the system. The discrepancies with the biology could result from the limitations of the data, numerical error, or the absence of key components in the system of equations. Even with the inconsistencies, our model can improve by incorporating more data, more significant variables, or even by simply modifying the equations to better imitate the biological interactions. While the model is currently unrefined, it has the potential to grow and develop into a future work benefiting research on obesity, chronic inflammation, and type 2 diabetes mellitus. 


\section{Acknowledgements}

The authors are grateful for support provided by the National Science Foundation and the National Institutes of Health under grants:

- NSF Award \#0755322 REU Site: Modeling and simulation of biological networks

- NIH/NSF Award \#0609225 Summer Institute for Quantitative and Integrative Bioengineering

- NCCAM at NIH Award \# 5R01AT4308

Additional support was provided by the Nutritional Immunology Group at the Virginia Bioinformatics Institute, the Virginia Bioinformatics Institute, and the Interdisciplinary Center for Applied Mathematics at Virginia Tech. We would also like to thank Drs. Josep Bassaganya-Riera and Amir Guri for providing the data and biological insight necessary to perform the research. We would like to thank Christy Koelling and Drs. Jeff Borggaard, John Burns, Eugene Cliff, Susan Faulkner, Raquel Hontecillas-Magarzo, Abdul Jarrah, Reinhard Laubenbacher, Henning Mortveit, and Lizette Zietsman for their contributions to discussions and oversight of the research. In addition, we would like to thank The MathWorks ${ }^{\mathrm{TM}}$ for providing software through their academic support program.

\section{References}

[1] J. BASSAGANYA-RIERA, Introduction to immunology lecture. June 2008.

[2] __, Obesity-related inflammation lecture. June 2008.

[3] B. De Blasio, P. BaK, F. Pociot, A. Karlsen, And J. Nerup, Onset of type 1 diabetes a dynamical instability, Diabetes, 48 (1999), pp. 1677-1685.

[4] P. DÍAz AND J. PÉREZ, Average adipocyte area and its relationship to body weight in db/db high-fat-diet obese mice. July 2008.

[5] D. Galuska, C. Gillespie, S. Kuester, A. Mokdad, M. Cogswell, And C. PHILIP, State-specific prevalence of obesity among adults-United States, 2007, Morbidity and Mortality Weekly Report, 57 (2008), pp. 765-768.

[6] A. Guri, R. Hontecillas, G. Ferrer, O. Casagran, U. Wankahade, A. NoBLe, D. Eizirik, F. ORTis, M. CNOP, D. LiU, H. Si, AND J. BAssaganya-Riera, Loss of PPAR $\gamma$ in immune cells impairs the ability of abscisic acid to improve insulin sensitivity by suppressing monocyte chemoattractant protein-1 expression and macrophage infiltration into white adipose tissue, Journal of Nutritional Biochemistry, 19 (2008), pp. 216228.

[7] A. Guri, R. Hontecillas, H. Si, D. LiU, And J. BassaganyA-RierA, Dietary abscisic acid ameliorates glucose tolerance and obesity-related inflammation in $\mathrm{db} / \mathrm{db}$ mice fed high-fat diets, Clinical Nutrition, 26 (2007), pp. 107-116. 
[8] G. LedDer, Differential Equations: A Modeling Approach, McGraw-Hill, 2005. Theorem 6.5.3.

[9] A. Maree, R. Kublik, D. Finegood, And L. Edelstein-Keshet, Modelling the onset of type 1 diabetes: can impaired macrophage phagocytosis make the difference between health and disease?, Philisopical Transactions of the Royal Society A, 364 (2006), pp. 1267-1282.

[10] A. Mokdad, B. Bowman, E. Ford, F. Vinicor, J. Marks, And J. Koplan, The continuing epidemics of obesity and diabetes in the United States, Journal of the American Medical Association, 286 (2001), pp. 1195-1200.

[11] B. POPKIN AND C. DOAK, The obesity epidemic is a worldwide phenomenon, Nutrition Review, 56 (1998), pp. 106-114.

[12] P. PusKa, C. NISHIDA, AND D. PORTER, World health organization strategy on diet, physical activity, and health: Obesity and overweight. Data and statistics. WHO, 2007.

[13] S. SHOELSON, J. LEe, AND A. GOLDFINE, Inflammation and insulin resistance, Journal of Clinical Investigation, 116 (2006), pp. 1793-1801.

[14] Y. Vodovotz, C. Chow, J. Bartels, C. Lagoa, J. Prince, R. Levy, R. Kumar, J. Day, J. Rubin, G. Constantine, T. Billiar, M. Fink, And G. Clermont, In silico models of acute inflammation in animals, Shock, 26 (2006), pp. 235-244.

[15] H. Xu, G. Barnes, G. T. Q. Yang, D. Yang, C. Chou, J. Sole, A. Nichols, J. Ross, L. TARTAGLIA, AND H. CHEN, Chronic inflammation in fat plays a crucial role in the development of obesity-related insulin resistance, Journal of Clinical Investigation, 112 (2003), pp. 1821-1830.

[16] S. YONA AND S. GORDON, Inflammation: Glucocorticoids turn the monocyte switch, Immunology and Cell Biology, 85 (2007), pp. 81-82. 